\title{
The impact of decompression with instrumentation on local failure following spine stereotactic radiosurgery
}

\author{
Jacob A. Miller, BS, ${ }^{1}$ Ehsan H. Balagamwala, MD, ${ }^{2}$ Camille A. Berriochoa, MD, ${ }^{2}$ \\ Lilyana Angelov, MD, $, 1,3,4$ John H. Suh, MD, ${ }^{1-3}$ Edward C. Benzel, MD, 1,4 \\ Alireza M. Mohammadi, MD, ${ }^{1,3,4}$ Todd Emch, MD, ${ }^{1,5}$ Anthony Magnelli, MS, ${ }^{2}$ \\ Andrew Godley, PhD, ${ }^{2}$ Peng Qi, PhD, ${ }^{2}$ and Samuel T. Chao, MD ${ }^{1-3}$
}

\begin{abstract}
${ }^{1}$ Cleveland Clinic Lerner College of Medicine of Case Western Reserve University, Cleveland Clinic; ${ }^{2}$ Department of Radiation Oncology, Taussig Cancer Institute, Cleveland Clinic; ${ }^{3}$ Rose Ella Burkhardt Brain Tumor and Neuro-Oncology Center, Taussig Cancer Institute, Cleveland Clinic; ${ }^{4}$ Department of Neurosurgery, Neurological Institute, Cleveland Clinic; and ${ }^{5}$ Department of Diagnostic Radiology, Imaging Institute, Cleveland Clinic, Cleveland, Ohio
\end{abstract}

\begin{abstract}
OBJECTIVE Spine stereotactic radiosurgery (SRS) is a safe and effective treatment for spinal metastases. However, it is unknown whether this highly conformal radiation technique is suitable at instrumented sites given the potential for microscopic disease seeding. The authors hypothesized that spinal decompression with instrumentation is not associated with increased local failure (LF) following SRS.
\end{abstract}

METHODS A 2:1 propensity-matched retrospective cohort study of patients undergoing SRS for spinal metastasis was conducted. Patients with less than 1 month of radiographic follow-up were excluded. Each SRS treatment with spinal decompression and instrumentation was propensity matched to 2 controls without decompression or instrumentation on the basis of demographic, disease-related, dosimetric, and treatment-site characteristics. Standardized differences were used to assess for balance between matched cohorts.

The primary outcome was the 12-month cumulative incidence of LF, with death as a competing risk. Lesions demonstrating any in-field progression were considered LFs. Secondary outcomes of interest were post-SRS pain flare, vertebral compression fracture, instrumentation failure, and any Grade $\geq 3$ toxicity. Cumulative incidences analysis was used to estimate LF in each cohort, which were compared via Gray's test. Multivariate competing-risks regression was then used to adjust for prespecified covariates.

RESULTS Of 650 candidates for the control group, 166 were propensity matched to 83 patients with instrumentation. Baseline characteristics were well balanced. The median prescription dose was 16 Gy in each cohort. The 12-month cumulative incidence of LF was not statistically significantly different between cohorts ( $22.8 \%$ [instrumentation] vs $15.8 \%$ [control], $p=0.25)$. After adjusting for the prespecified covariates in a multivariate competing-risks model, decompression with instrumentation did not contribute to a greater risk of LF (HR 1.21, 95\% Cl 0.74-1.98, $p=0.45$ ). The incidences of post-SRS pain flare ( $11 \%$ vs $14 \%, p=0.55)$, vertebral compression fracture $(12 \%$ vs $22 \%, p=0.04)$, and Grade $\geq 3$ toxicity $(1 \%$ vs $1 \%, p=1.00)$ were not increased at instrumented sites. No instrumentation failures were observed.

CONCLUSIONS In this propensity-matched analysis, LF and toxicity were similar among cohorts, suggesting that decompression with instrumentation does not significantly impact the efficacy or safety of spine SRS. Accordingly, spinal instrumentation may not be a contraindication to SRS. Future studies comparing SRS to conventional radiotherapy at instrumented sites in matched populations are warranted.

https://thejns.org/doi/abs/10.3171/2017.3.SPINE161015

KEY WORDS spine metastasis; stereotactic radiosurgery; spinal instrumentation; local failure; oncology

ABBREVIATIONS KPS = Karnofsky Performance Scale; LF = local failure; NFS = neurological function scale; OS = overall survival; PTV = planning treatment volume; SRS = stereotactic radiosurgery. 
$\mathrm{R}$ ADIATION therapy and oncological resection with stabilization are the primary modalities for the management of gross spinal metastatic disease. For patients with metastatic spinal cord compression, randomized data have demonstrated superior functional outcomes following decompression with postoperative radiotherapy compared with radiotherapy alone..$^{14,15,18}$ For patients without spinal cord compression or emergency spinal instability, radiotherapy alone offers palliation, local control, and prophylaxis against cord compression without the morbidity and cost of surgery. In an effort to provide dose escalation while sparing organs at risk, highly conformal radiation techniques have been developed. Spine stereotactic radiosurgery (SRS) is increasingly being used in the management of recurrent vertebral metastasis, radioresistant disease, and oligometastatic disease. ${ }^{4}$ Although no randomized data have been reported, many retrospective studies suggest that spine SRS offers superior local control relative to conventional radiotherapy. ${ }^{2,3,8}$

Patients with spinal instability or cord compression are specifically excluded from the Radiation Therapy Oncology Group (RTOG) study 0631, which is currently randomizing patients to single-fraction SRS or single-fraction conventional radiotherapy in an effort to better evaluate the benefits of SRS..$^{19}$ In the postoperative setting, small retrospective studies have suggested that SRS is safe and effective. ${ }^{5,12,16}$ These studies have generally been noncomparative, and therefore the ability to draw meaningful conclusions is limited. In particular, it is unclear whether SRS is safe and effective at instrumented sites given the possibility of microscopic disease seeding at the time of decompression and instrumentation. Although greater conformality may offer spinal cord sparing, local failure (LF) at instrumented sites may be increased. We hypothesized that decompression with instrumentation is not associated with a greater cumulative incidence of LF following SRS, compared with SRS at surgically naïve sites.

\section{Methods}

\section{Patient Selection and Data Collection}

An institutional review board-approved, retrospective, 2:1 propensity-matched cohort study was conducted to compare patients with and without decompression on receiving instrumentation. All patients undergoing spine SRS for either multiple myeloma or solid-tumor metastasis between 2006 and 2014 were eligible for inclusion. Treatments with less than 1 month of radiographic followup were excluded. Indications for radiosurgery were either related to symptoms (pain, neurological deficit) or radiographic progression (control of primary tumor or epidural disease). Patients with rapidly progressive neurological deficits or emergency spinal instability were not eligible for SRS alone. However, patients were not excluded on the basis of epidural disease or number of involved vertebrae. The instrumentation cohort comprised all treatment sites in patients with any instrumentation following decompression, including pedicle screws, plates, interbody cages, or fixation rods. Treatment sites with prior surgical decompression without fixation or vertebral augmentation alone were not included in the instrumentation cohort and were not eligible for the control cohort.

\section{Radiosurgical Treatment}

The SRS was conducted as described previously.,17 Briefly, treatment planning used CT simulation with 1.5$\mathrm{mm}$ slice thickness fused to a high-definition MRI. The $\mathrm{CT}$ and MRI fusion was performed in BrainScan or iPlan software (Brainlab) or MIM (MIM Software, Inc.). In general, target volumes were contoured according to the International Spine Radiosurgery Consortium Consensus Guidelines. ${ }^{6}$ At instrumented sites, the gross tumor volume was the residual disease extent on the postoperative MRI, whereas the clinical target volume contained the preoperative disease extent, as well as adjacent bony compartments that were at risk for disease extension. The spinal cord or cauda equina planning risk volume was contoured approximately $5 \mathrm{~mm}$ above and below the target volume. For patients with significant instrumentation artifact, CT myelograms were obtained to define normal anatomy. The spinal cord volume was constrained to $\mathrm{V} 10 \mathrm{~Gy}<10 \%$ and a maximum point dose of 14 Gy for single-fraction SRS. The cauda equina was constrained to V12 Gy $<10 \%$ and a maximum point dose of $16 \mathrm{~Gy}$. The most common singlefraction prescription dose was 16 Gy (range 10-18 Gy), whereas hypofractionated regimens included 21 or 24 Gy in 3 fractions. Image guidance was achieved with conebeam CT and/or the ExacTrac system (Brainlab). At 6-8 weeks following SRS, patients were seen in clinic after spine MRI or CT myelograms were obtained and then followed every 3 months thereafter.

\section{Primary and Secondary Outcomes}

The primary outcome was the cumulative incidence of LF, whereas secondary outcomes included overall survival (OS) and the incidences of post-SRS pain flare, vertebral fracture, instrumentation failure, and any Grade $\geq$ 3 toxicity. Lesions demonstrating any in-field progression as assessed by radiation oncologists, neurosurgeons, and/ or neuroradiologists were considered LFs. Patients experiencing a transient increase in pain responsive to steroids within 14 days of SRS were considered to have a pain flare. Radiographic evidence of de novo or progressed vertebral body collapse was considered a vertebral compression fracture. These practices were standard throughout the study period. All outcomes were calculated from the date of SRS completion.

\section{Statistical Analysis}

Each SRS treatment with in-field decompression and instrumentation was propensity matched to 2 controls without decompression and instrumentation on the basis of demographic, disease-related, dosimetric, and treatment-site characteristics. An optimal matching algorithm was used. ${ }^{11}$ Standardized differences were used to assess balance between matched cohorts. ${ }^{21}$ Data were analyzed on a per-treatment basis. Baseline continuous data were compared with Wilcoxon rank-sum tests, whereas categorical data were compared with Fisher exact tests. The Kaplan-Meier method was used to determine estimates of OS, which were compared with log-rank tests. Based on the subdistribution method, cumulative incidence analysis was used to determine estimates of LF, with death as a competing risk. ${ }^{10}$ Noninformative censoring was per- 
formed at loss to radiographic follow-up. Differences in cumulative incidences were compared via Gray's tests. ${ }^{10}$ Based on the method of Fine and Gray, multivariate competing-risks regression was used to model LF, with death as a competing risk. ${ }^{7}$ Models were adjusted for the following covariates: age; sex; smoking status; neurological function scale (NFS) score; Karnofsky Performance Scale (KPS) score; number of prior chemotherapy regimens; tumor pathology; systemic disease status; extent of spinal disease; treatment site location; number of treated vertebrae; presence of epidural, neural foraminal, paraspinal, or posterior elements disease; any prior in-field irradiation; and dosimetric characteristics. Covariates demonstrating an association ( $\mathrm{p} \leq 0.20)$ with LF on univariate analysis were evaluated in a multivariate model including all 2-way interactions. Covariate inclusion into the final model required $\mathrm{p} \leq 0.10$. Analyses were conducted using the $\mathrm{R}$ "cmprsk" statistical software package. Two-sided tests with $\mathrm{p}<0.05$ were considered statistically significant.

\section{Results}

\section{Patient and Treatment Characteristics}

Of 650 candidates for the control group, 166 were propensity matched to 83 patients with instrumentation. Excellent balance (standardized difference $\leq 0.10$ ) was achieved for nearly all characteristics between cohorts (Table 1). Characteristics with standardized differences exceeding an absolute value of 0.10 included the following: KPS score (median 80 vs $80, \mathrm{p}=0.17$ ); NFS score (median 1 vs $1, \mathrm{p}=0.12$ ); radioresistant $(39 \%$ vs $33 \%, \mathrm{p}=$ $0.73)$ and lung $(12 \%$ vs $19 \%, \mathrm{p}=0.73)$ histological types; presence of extraspinal bone metastases ( $43 \%$ vs $50 \%$, p $=0.35)$; pre-SRS vertebral body fracture $(35 \%$ vs $28 \%$, p $=0.31$ ); maximum dose (mean 18.2 vs $17.6 \mathrm{~Gy}, \mathrm{p}=0.24$ ); planning treatment volume (PTV) (mean 115.9 vs 101.5 $\mathrm{cm}^{3}, \mathrm{p}=0.22$ ); and the conformality index (mean $1.3 \mathrm{vs}$ $1.4, \mathrm{p}=0.11$ ). No statistically significant differences in baseline characteristics were observed.

The median time from spinal instrumentation to SRS was 1.7 months. Posterior-only approaches (51 sites, 61\%) were more common than anterior (17 sites, $21 \%$ ) or combined anterior-posterior (15 sites, 18\%) approaches. Corpectomy with cage or strut placement was performed at 48 sites (58\%). All patients underwent spinal fixation with screws and either plates or rods.

In each cohort, the median prescription dose was $16 \mathrm{~Gy}$ in a single fraction. Patients were followed radiographically for a median of 9 months (range 1-87 months) in the instrumentation cohort and 6 months (range 1-86 months) in the control cohort ( $\mathrm{p}=0.18$ ). The median OS was 18 months among patients in the instrumentation cohort (95\% CI 12-29 months), compared with 12 months in the control cohort (95\% CI 9-19 months; $\mathrm{p}=0.17$, Fig. 1). In a comparison between the instrumentation and control cohorts, the 12-month cumulative incidences of LF were $22.8 \%$ (95\% CI $13.4 \%-32.1 \%$ ) and $15.8 \%$ (95\% CI $10.0 \%-21.5 \%)$, respectively $(\mathrm{p}=0.25$; Fig. 2 , Table 2 ). The incidences of in-field salvage surgery $(7 \%$ vs $4 \%, p=$ 0.37 ) and reirradiation ( $16 \%$ vs $12 \%, \mathrm{p}=0.43$ ) did not significantly differ between cohorts. Furthermore, we found fewer incidences of pain flare $(11 \%$ vs $14 \%, \mathrm{p}=0.55)$ and post-SRS vertebral fracture ( $12 \%$ vs $22 \%, \mathrm{p}=0.04)$, and no difference in Grade $\geq 3$ toxicity $(1 \%$ vs $1 \%, \mathrm{p}=1.00)$ at instrumented sites. No instrumentation failures were observed after SRS.

Multivariate competing-risks regression was then used to adjust for measured differences between cohorts. Several covariates were associated ( $p \leq 0.20)$ with LF on univariate analysis and were evaluated alongside spinal instrumentation in a multivariate model (Table 3). Multivariate analysis demonstrated that controlled systemic disease (HR 1.72, 95\% CI 1.06-2.78, $\mathrm{p}=0.03$ ), PTV coverage (HR 0.89, 95\% CI 0.81-0.99, $\mathrm{p}=0.03$ ), and PTV (HR 1.01, 95\% CI 1.01-1.03, $\mathrm{p}=0.02$ ) were significantly associated with LF. However, the presence of spinal instrumentation was not significantly associated with LF following multivariate analysis (HR $1.21,95 \%$ CI 0.74 $1.98, \mathrm{p}=0.45)$.

\section{Discussion}

The use of highly conformal radiation therapy at instrumented sites is controversial secondary to concerns for intraoperative seeding that may increase the risk for LF. Given a lack of comparative data in the literature, we performed a propensity-matched retrospective cohort study. We hypothesized that decompression with instrumentation would not significantly increase the cumulative incidence of LF. With respect to measured confounders, matching achieved comparable cohorts with very similar baseline characteristics. Following multivariate competing-risks regression, a statistically significant increase in the rate of LF attributable to decompression with instrumentation was not observed (HR 1.21, 95\% CI 0.74-1.98, $\mathrm{p}=0.45$ ).

Several retrospective case series and cohort studies have reported local control following SRS at sites of surgical decompression, without an emphasis on instrumentation. In 2013, Laufer et al. ${ }^{16}$ reported a noncomparative series of 186 patients who underwent posterior surgical decompression followed by SRS. The median follow-up was 8 months, and the 12-month cumulative incidence of LF was $16.4 \%$ (comparable to the present investigation). Patients undergoing high-dose hypofractionated SRS (2430 Gy in 3 fractions) or single-fraction SRS (24 Gy) had a lower cumulative incidence of LF relative to low-dose hypofractionated SRS (18-36 Gy in 5 or 6 fractions) (4.1\% and $9.0 \%$ vs $22.2 \%, p=0.04$ ). Although this offers insight into useful fractionation schemes, this investigation does not offer a comparison between instrumented and noninstrumented sites. However, this relatively large investigation did offer a precedent for epidural decompression with adjuvant SRS as a possible alternative to corpectomy with stabilization.

In a comparative retrospective cohort study reported by Bate et al., 48 treatment sites underwent SRS alone, while 21 underwent epidural decompression with adjuvant SRS. ${ }^{5}$ No matching or multivariate methods were used in an attempt to control for differences between cohorts. The median follow-up was 10 months, and the 12-month cumulative incidences of LF were $4.2 \%$ (SRS alone) and 
TABLE 1. Baseline characteristics of matched populations of patients with spinal metastases

\begin{tabular}{|c|c|c|c|c|}
\hline Characteristic & Instrumentation Group & Control Group & Standardized Diff & $\mathrm{p}$ Value \\
\hline No. of treatments & 83 & 166 & & \\
\hline \multicolumn{5}{|l|}{ Patient characteristics } \\
\hline Age (yrs) & $60.6 \pm 11.9$ & $60.6 \pm 12.8$ & 0.00 & 0.99 \\
\hline Sex: male & $52(63)$ & $103(62)$ & 0.00 & 1.00 \\
\hline Race: Caucasian & $72(87)$ & $141(85)$ & 0.05 & 0.85 \\
\hline Prior smoker & $49(59)$ & $99(60)$ & -0.01 & 1.00 \\
\hline Current smoker & $8(10)$ & $14(8)$ & 0.04 & 0.81 \\
\hline KPS score & $80[60-100]$ & $80[60-100]$ & -0.20 & 0.17 \\
\hline NFS score & $1[0-4]$ & $1[0-4]$ & 0.19 & 0.12 \\
\hline $\mathrm{BMI}$ & $27.2 \pm 5.7$ & $27.2 \pm 5.2$ & 0.01 & 0.95 \\
\hline Diabetes & $10(12)$ & $21(13)$ & -0.02 & 1.00 \\
\hline No. of prior chemo regimens & $1[0-4]$ & $1[0-3]$ & -0.01 & 0.15 \\
\hline \multicolumn{5}{|l|}{ Disease characteristics } \\
\hline Primary pathology & & & & 0.73 \\
\hline Radioresistant* & $32(39)$ & $54(33)$ & 0.12 & \\
\hline Other & $25(30)$ & $43(26)$ & 0.09 & \\
\hline Lung & $10(12)$ & $31(19)$ & -0.20 & \\
\hline Breast & $7(8)$ & $17(10)$ & -0.06 & \\
\hline Prostate & $5(6)$ & $11(7)$ & -0.03 & \\
\hline Radiosensitive & $4(5)$ & $10(6)$ & -0.06 & \\
\hline Controlled primary & $47(57)$ & $102(61)$ & -0.10 & 0.49 \\
\hline Controlled systemic disease & $38(46)$ & $83(50)$ & -0.06 & 0.59 \\
\hline Visceral metastases & $34(41)$ & $73(44)$ & -0.06 & 0.69 \\
\hline Extraspinal bone metastases & $36(43)$ & $83(50)$ & -0.13 & 0.35 \\
\hline Spinal disease burden & & & & 0.90 \\
\hline Single level & $30(36)$ & $58(35)$ & 0.02 & \\
\hline Multilevel (2-5 vertebrae) & $41(49)$ & $80(48)$ & 0.02 & \\
\hline Diffuse (>5 vertebrae) & $12(15)$ & $28(17)$ & 0.07 & \\
\hline \multicolumn{5}{|l|}{ Treatment site characteristics } \\
\hline Location & & & & 0.68 \\
\hline Cervical & $18(22)$ & $30(18)$ & 0.09 & \\
\hline Thoracic & $47(57)$ & $92(55)$ & 0.01 & \\
\hline Lumbosacral & $18(22)$ & $43(26)$ & -0.10 & \\
\hline No. of treated vertebrae & $1[0-5]$ & $1[0-6]$ & 0.08 & 0.39 \\
\hline Vertebral body disease & $80(96)$ & $159(96)$ & 0.03 & 1.00 \\
\hline Epidural disease & $73(88)$ & $142(86)$ & 0.07 & 0.70 \\
\hline Neural foramen disease & $59(71)$ & $120(72)$ & -0.03 & 0.88 \\
\hline Paraspinal disease & $42(51)$ & $81(49)$ & 0.04 & 0.79 \\
\hline Posterior elements disease & $40(48)$ & $83(50)$ & -0.04 & 0.89 \\
\hline Vertebral body fracture & $29(35)$ & $47(28)$ & 0.14 & 0.31 \\
\hline Prior radiotherapy & $34(41)$ & $60(36)$ & -0.01 & 0.58 \\
\hline \multicolumn{5}{|l|}{ Dosimetric characteristics } \\
\hline Fractions & $1[0-5]$ & $1[0-5]$ & 0.06 & 0.47 \\
\hline Prescription dose (Gy) & $15.6 \pm 2.7$ & $15.3 \pm 2.1$ & 0.10 & 0.46 \\
\hline Maximum dose (Gy) & $18.2 \pm 3.6$ & $17.6 \pm 2.7$ & 0.13 & 0.24 \\
\hline Minimum dose (Gy) & $10.2 \pm 2.4$ & $10.0 \pm 1.9$ & 0.07 & 0.93 \\
\hline PTV coverage (\%) & $94.0 \pm 2.5$ & $93.9 \pm 2.7$ & 0.03 & 0.82 \\
\hline PTV $\left(\mathrm{cm}^{3}\right)$ & $115.9 \pm 112.2$ & $101.5 \pm 114.8$ & 0.13 & 0.22 \\
\hline Maximum diam (cm) & $7.7 \pm 2.6$ & $7.1 \pm 2.7$ & 0.15 & 0.28 \\
\hline
\end{tabular}


» CONTINUED FROM PAGE 439

TABLE 1. Baseline characteristics of matched populations of patients with spinal metastases

\begin{tabular}{ccccc}
\hline Characteristic & Instrumentation Group & Control Group & Standardized Diff & $p$ Value \\
\hline Dosimetric characteristics (continued) & & & & \\
\hline Conformality index & $1.3 \pm 0.2$ & $1.4 \pm 0.4$ & -0.15 & 0.11 \\
\hline Heterogeneity index & $1.2 \pm 0.1$ & $1.2 \pm 0.1$ & 0.00 & 0.93 \\
\hline Radiographic follow-up (mos) & $14.9 \pm 17.8$ & $14.0 \pm 19.2$ & 0.05 & 0.18 \\
\hline
\end{tabular}

$\mathrm{BMI}=$ body mass index; chemo = chemotherapy; diam = diameter; diff = difference .

Values are presented as the mean $\pm \mathrm{SD}$, median [range], or number $(\%)$.

* Melanoma, sarcoma, or renal cell carcinoma.

9.5\% (SRS + surgery), without consideration of competing risks. The authors concluded that SRS with or without epidural decompression represents an effective alternative to radical oncological resection. However, this conclusion was an extrapolation, because no comparison cohort of radical oncological resection was included. Given the small sample size and lack of adjustment for confounding, it is difficult to draw meaningful conclusions from these data.

Beyond these concerns for LF, there is some evidence suggesting that SRS may decrease the rate of instrumentation failure relative to conventional radiotherapy. In a small retrospective cohort study of 15 patients, Harel and Zach reported that the risk of instrumentation failure was 43\% among patients undergoing conventional radiotherapy, compared with $0 \%$ among patients undergoing SRS (p $=0.08) .{ }^{13}$ This small sample was not matched or adjusted to account for confounding. Furthermore, patients in the conventionally fractionated radiotherapy cohort received doses usually reserved for definitive courses (>30 Gy). In general, patients undergoing conventional radiotherapy probably represent a dissimilar population relative to patients undergoing SRS. Future studies might compare these 2 techniques at instrumented sites in an effort to determine the risk-benefit profile of dose escalation and greater conformality. Since this publication, a larger multiinstitutional series of 318 patients reported a low inci- dence $(2.8 \%)$ of instrumentation failure following spine SRS. ${ }^{1}$ Without a matched comparator arm, it is unknown whether a similarly low incidence would be observed following conventional radiotherapy.

Our findings must be carefully interpreted in the context of competing risks, because median survival was nonsignificantly greater in the instrumentation cohort (18 vs 12 months, $p=0.17$, Fig. 1). This could be interpreted as evidence of a survival benefit with the use of decompression and fixation, analogous to the survival benefit reported by Patchell et al. in a randomized trial of circumferential decompression with radiotherapy versus radiotherapy alone. ${ }^{18}$ Alternatively, the observed survival difference may be evidence of unmeasured confounding despite propensity matching across more than 30 covariates. Certainly, patients must be medically fit to undergo surgery, thereby biasing survival in favor of the instrumentation cohort. Although the 12-month cumulative incidence of LF was not statistically significantly different $(22.8 \%$ vs $15.8 \%$, $\mathrm{p}=0.25$, Fig. 2), a larger multiinstitutional investigation might detect a significant difference. This difference in LF may be due to differences in survival; the 12-month cumulative incidence of either LF or death was $47.4 \%$ (95\% CI $36.1 \%-58.6 \%)$ in the instrumentation cohort, compared with $58.4 \%$ (95\% CI 50.6\%-66.2\%, p = 0.10) among patients in the control cohort. Therefore, extended survival among patients with instrumentation may be responsible

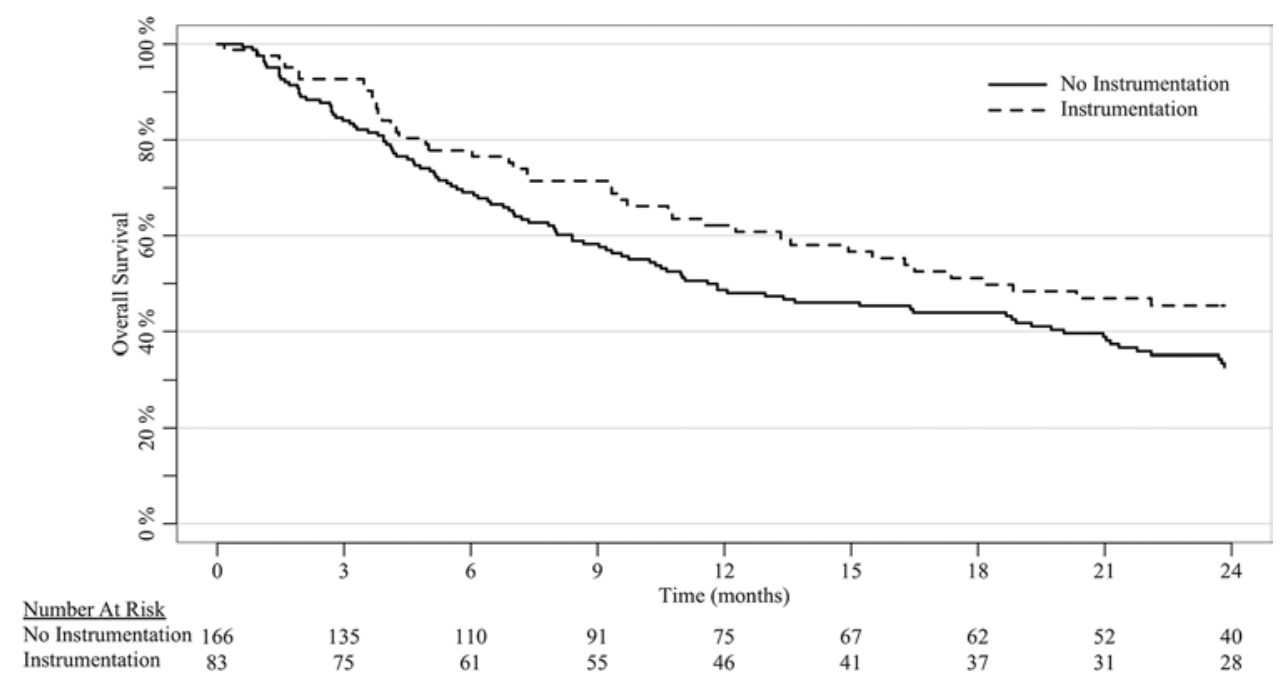

FIG. 1. Graph showing OS following SRS with and without in-field spinal instrumentation. 


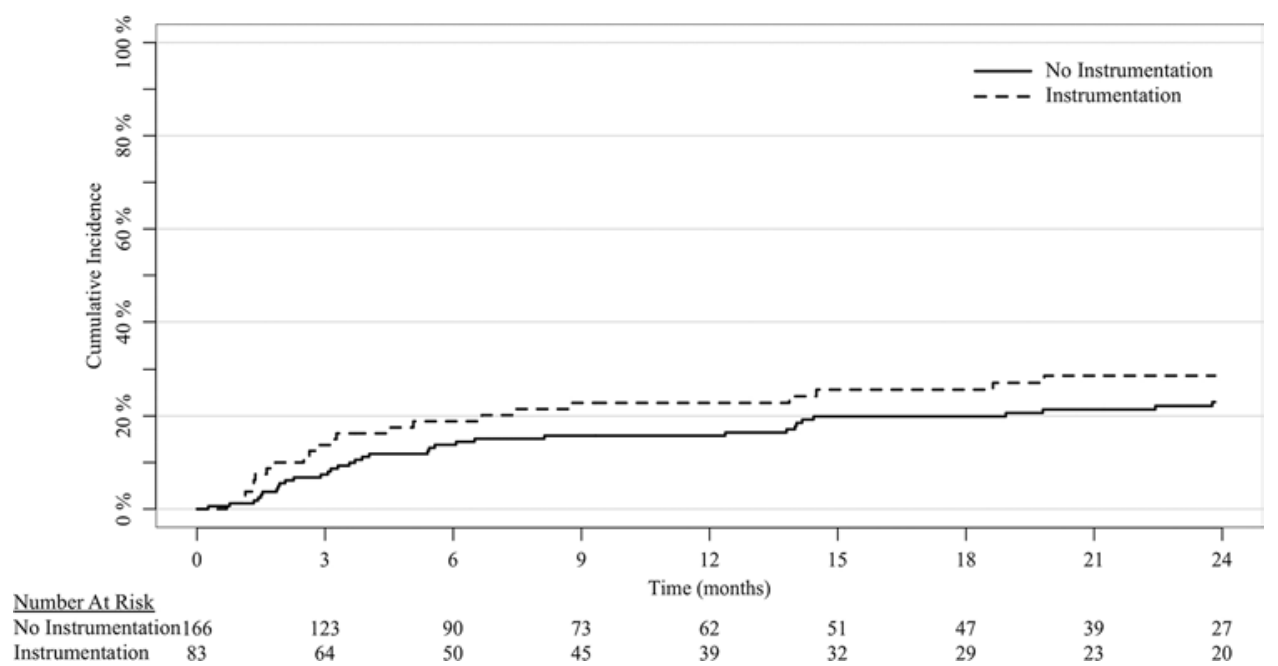

FIG. 2. Graph showing the cumulative incidence of LF following SRS with and without in-field spinal instrumentation.

for the absolute (albeit small) difference in LF, rather than the effect of decompression with instrumentation itself. Similarly, patients with controlled systemic disease were at greater risk for LF (HR 1.72, 95\% CI 1.06-2.78, p = 0.03 ), probably due to extended survival in this population. Randomizing patients with spinal instability to SRS alone versus SRS with instrumentation would be unethical, and therefore this retrospective comparative investigation with rigorous matching is necessary to control for sources of confounding.

\section{Strengths and Limitations}

Several limitations must be considered when interpreting the results of this study. Patients undergoing spinal decompression with instrumentation are inherently different from patients whose disease is managed with SRS alone, introducing selection bias. These patients are more likely to have extensive epidural disease, cord compression, or bony retropulsion compared with patients undergoing SRS alone; although the extent of epidural disease is not explicitly graded at our institution, it is encouraging that similar rates of LF were observed between cohorts despite a greater expected degree of epidural disease in the instrumentation cohort. This may reflect superior dosimetric coverage of the epidural space following surgical decompression. Although cohorts were propensity matched across more than 30 covariates, unmeasured confounding potentially limits the internal validity of this investigation. However, randomization would be necessary to discern the independent effect of spinal fixation on survival and LF. A further limitation is the precision (or lack thereof) of MRI at instrumented sites, which may have increased the time to detection of LF. Alternatively, patients with instrumentation may undergo imaging more frequently, increasing the

TABLE 2. Unadjusted clinical outcomes in patients with spinal metastases

\begin{tabular}{lccc}
\hline \multicolumn{1}{c}{ Characteristic } & Instrumentation & Control & p Value \\
\hline No. of treatments & 83 & 166 & 0.17 \\
\hline Median OS in mos & $18[12-29]$ & $12[9-19]$ & 0.25 \\
\hline Cumulative incidence of LF & & & \\
\hline 6 mos & $18.8 \%[10.1-27.5 \%]$ & $13.8 \%[9.2-18.9 \%]$ & 0.37 \\
\hline 12 mos & $22.8 \%[13.4-32.1 \%]$ & $15.8 \%[10.0-21.5 \%]$ & 0.43 \\
\hline Failure requiring surgery & $6(7)$ & $7(4)$ & 0.55 \\
\hline Failure requiring reirradiation & $13(16)$ & $20(12)$ & 0.04 \\
\hline Adverse events & & & 1.00 \\
\hline Pain flare & $9(11)$ & $23(14)$ & 0.33 \\
\hline Vertebral fracture & $10(12)$ & $37(22)$ & 1.00 \\
\hline Grade $\geq 3$ toxicity & $1(1)$ & $1(1)$ & $\mathrm{NA}$ \\
\hline Esophagitis & $1(1)$ & $0(0)$ & $1(1)$ \\
\hline Myelopathy & $0(0)$ & $\mathrm{NA}$ & \\
\hline Instrumentation failure & $0(0)$ & & \\
\hline
\end{tabular}

$\mathrm{NA}=$ not applicable.

Values are presented as estimate $[95 \% \mathrm{Cl}]$ or number $(\%)$. 
TABLE 3. Multivariate competing-risks regression for LF in patients with spinal metastases

\begin{tabular}{|c|c|c|c|c|}
\hline \multirow[b]{2}{*}{ Covariate } & \multirow{2}{*}{$\begin{array}{c}\text { Univariate, } \\
\text { p Value }\end{array}$} & \multicolumn{3}{|c|}{ Multivariate } \\
\hline & & $\mathrm{HR}$ & $95 \% \mathrm{Cl}$ & p Value \\
\hline Spinal instrumentation & 0.30 & 1.21 & $0.74-1.98$ & 0.45 \\
\hline \multicolumn{5}{|l|}{ Patient characteristics } \\
\hline Age (yrs) & 0.90 & - & - & - \\
\hline Prior smoker & 0.29 & - & - & - \\
\hline Current smoker & 0.90 & - & - & - \\
\hline KPS score & 0.98 & - & - & - \\
\hline NFS score & 0.12 & 0.73 & $0.56-1.06$ & 0.06 \\
\hline Diabetes & 0.03 & 1.59 & $0.87-2.91$ & 0.13 \\
\hline No. of prior chemo regimens & 0.26 & - & - & - \\
\hline \multicolumn{5}{|l|}{ Disease characteristics } \\
\hline \multicolumn{5}{|l|}{ Primary pathology } \\
\hline Radioresistant* & 0.04 & 2.41 & $0.94-6.13$ & 0.07 \\
\hline Other & 0.03 & 2.95 & $0.93-7.69$ & 0.07 \\
\hline Lung & Reference & 1.00 & Reference & Reference \\
\hline Breast & 0.55 & 1.40 & $0.41-4.77$ & 0.70 \\
\hline Prostate & 0.45 & 1.49 & $0.41-5.47$ & 0.55 \\
\hline Radiosensitive & 0.83 & 0.79 & $0.16-3.80$ & 0.76 \\
\hline Controlled primary disease & 0.83 & - & - & - \\
\hline Controlled systemic disease & 0.11 & 1.72 & $1.06-2.78$ & 0.03 \\
\hline Visceral metastases & 0.41 & - & - & - \\
\hline Extraspinal bone metastases & 0.54 & - & - & - \\
\hline Spinal disease burden & & & & - \\
\hline Single level & Reference & - & - & - \\
\hline Multilevel (2-5 vertebrae) & 0.36 & - & - & - \\
\hline Diffuse (>5 vertebrae) & 0.87 & - & - & - \\
\hline \multicolumn{5}{|l|}{ Treatment site characteristics } \\
\hline \multicolumn{5}{|l|}{ Location } \\
\hline Cervical & Reference & 1.00 & Reference & Reference \\
\hline Thoracic & 0.11 & 1.49 & $0.58-3.34$ & 0.30 \\
\hline Lumbosacral & 0.22 & 1.40 & $0.71-3.14$ & 0.45 \\
\hline No. of treated vertebrae & 0.02 & 1.11 & $0.88-1.40$ & 0.39 \\
\hline Vertebral body & 0.80 & - & - & - \\
\hline Epidural disease & 0.15 & 1.39 & $0.59-3.25$ & 0.45 \\
\hline Neural foramen disease & 0.17 & 1.22 & $0.66-2.27$ & 0.52 \\
\hline Paraspinal disease & 0.43 & - & - & - \\
\hline Posterior elements disease & 0.85 & - & - & - \\
\hline Prior radiotherapy & 0.50 & - & - & - \\
\hline \multicolumn{5}{|l|}{ Dosimetric characteristics } \\
\hline Prescription dose (Gy) & 0.16 & 1.03 & $0.92-1.15$ & 0.61 \\
\hline Maximum dose (Gy) & 0.06 & 1.03 & $0.96-1.11$ & 0.40 \\
\hline Minimum dose (Gy) & 0.19 & 0.92 & $0.79-1.07$ & 0.25 \\
\hline PTV coverage (\%) & 0.04 & 0.89 & $0.81-0.99$ & 0.03 \\
\hline PTV $\left(\mathrm{cm}^{3}\right)^{*}$ & $<0.01$ & 1.01 & $1.01-1.03$ & 0.02 \\
\hline Maximum diam (cm) & 0.09 & 0.91 & $0.76-1.08$ & 0.27 \\
\hline Conformality index & 0.28 & - & - & - \\
\hline Heterogeneity index & 0.41 & - & - & - \\
\hline
\end{tabular}

$-=$ not calculated (variables did not meet the univariate $p$ value threshold and are not included in the final multivariate model).

* Per 10-unit increase. 
likelihood of earlier detection of LF. Moreover, titanium implants decrease the accuracy of dosimetry at instrumented sites. ${ }^{9,20}$ Use of propensity-matched controls and competing-risks analysis distinguishes the present study from prior investigations, which have generally been noncomparative or unmatched retrospective studies evaluating local control without consideration of competing risks.

\section{Conclusions}

In this propensity-matched analysis, LF and toxicity were similar among cohorts, suggesting that decompression with instrumentation does not significantly impact the efficacy or safety of spine SRS. Accordingly, spinal instrumentation may not be a contraindication to SRS. Future studies comparing SRS to conventional radiotherapy at instrumented sites in matched populations are warranted.

\section{References}

1. Amankulor NM, Xu R, Iorgulescu JB, Chapman T, Reiner AS, Riedel E, et al: The incidence and patterns of hardware failure after separation surgery in patients with spinal metastatic tumors. Spine J 14:1850-1859, 2014

2. Amdur RJ, Bennett J, Olivier K, Wallace A, Morris CG, Liu $\mathrm{C}$, et al: A prospective, phase II study demonstrating the potential value and limitation of radiosurgery for spine metastases. Am J Clin Oncol 32:515-520, 2009

3. Balagamwala EH, Angelov L, Koyfman SA, Suh JH, Reddy CA, Djemil T, et al: Single-fraction stereotactic body radiotherapy for spinal metastases from renal cell carcinoma. J Neurosurg Spine 17:556-564, 2012

4. Balagamwala EH, Cherian S, Angelov L, Suh JH, Djemil T, Lo SS, et al: Stereotactic body radiotherapy for the treatment of spinal metastases. J Radiat Oncol 1:255-265, 2012

5. Bate BG, Khan NR, Kimball BY, Gabrick K, Weaver J: Stereotactic radiosurgery for spinal metastases with or without separation surgery. J Neurosurg Spine 22:409-415, 2015

6. Cox BW, Spratt DE, Lovelock M, Bilsky MH, Lis E, Ryu S, et al: International Spine Radiosurgery Consortium consensus guidelines for target volume definition in spinal stereotactic radiosurgery. Int J Radiat Oncol Biol Phys 83:e597e605, 2012

7. Fine JP, Gray RJ: A proportional hazards model for the subdistribution of a competing risk. J Am Stat Assoc 94:496509, 1999

8. Gerszten PC, Burton SA, Ozhasoglu C, Welch WC: Radiosurgery for spinal metastases: clinical experience in 500 cases from a single institution. Spine (Phila Pa 1976) 32:193-199, 2007

9. Grams MP, Fong de Los Santos LE, Antolak JA, Brinkmann DH, Clarke MJ, Park SS, et al: Cadaveric verification of the Eclipse AAA algorithm for spine SBRT treatments with titanium hardware. Pract Radiat Oncol 6:131-141, 2016

10. Gray RJ: A class of K-sample tests for comparing the cumulative incidence of a competing risk. Ann Stat 16:1141-1154, 1988

11. Gu XS, Rosenbaum PR: Comparison of multivariate matching methods: structures, distances, and algorithms. J Comput Graph Stat 2:405-420, 1993

12. Harel R, Chao S, Krishnaney A, Emch T, Benzel EC, Angelov L: Spine instrumentation failure after spine tumor resection and radiation: comparing conventional radiotherapy with stereotactic radiosurgery outcomes. World Neurosurg 74:517-522, 2010

13. Harel R, Zach L: Spine radiosurgery for spinal metastases: indications, technique and outcome. Neurol Res 36:550-556, 2014

14. Kim JM, Losina E, Bono CM, Schoenfeld AJ, Collins JE, Katz JN, et al: Clinical outcome of metastatic spinal cord compression treated with surgical excision \pm radiation versus radiation therapy alone: a systematic review of literature. Spine (Phila Pa 1976) 37:78-84, 2012

15. Klimo P Jr, Thompson CJ, Kestle JRW, Schmidt MH: A meta-analysis of surgery versus conventional radiotherapy for the treatment of metastatic spinal epidural disease. Neuro Oncol 7:64-76, 2005

16. Laufer I, Iorgulescu JB, Chapman T, Lis E, Shi W, Zhang Z, et al: Local disease control for spinal metastases following "separation surgery" and adjuvant hypofractionated or highdose single-fraction stereotactic radiosurgery: outcome analysis in 186 patients. J Neurosurg Spine 18:207-214, 2013

17. Miller JA, Balagamwala EH, Angelov L, Suh JH, Rini B, Garcia JA, et al: Spine stereotactic radiosurgery with concurrent tyrosine kinase inhibitors for metastatic renal cell carcinoma. J Neurosurg Spine 25:766-774, 2016

18. Patchell RA, Tibbs PA, Regine WF, Payne R, Saris S, Kryscio RJ, et al: Direct decompressive surgical resection in the treatment of spinal cord compression caused by metastatic cancer: a randomised trial. Lancet 366:643-648, 2005

19. Ryu S, Pugh SL, Gerszten PC, Yin FF, Timmerman RD, Hitchcock YJ, et al: RTOG 0631 Phase II/III study of imageguided stereotactic radiosurgery for localized (1-3) spine metastases: phase II results. Int J Radiat Oncol Biol Phys 81:S131-S132, 2011

20. Wang X, Yang JN, Li X, Tailor R, Vassilliev O, Brown P, et al: Effect of spine hardware on small spinal stereotactic radiosurgery dosimetry. Phys Med Biol 58:6733-6747, 2013

21. Yang Y, Dalton JE: A unified approach to measuring the effect size between two groups using SAS $^{\circledR}$. SAS Global Forum. (http://support.sas.com/resources/papers/ proceedings12/335-2012.pdf ) [Accessed March 31, 2017]

\section{Disclosures}

Dr. Suh is a consultant for and received research support from Varian Medical Systems, and he received travel and speaker's fees from Elekta and Philips. Dr. Benzel has the following disclosures: royalties from Elsevier Publications and Thieme Publications; stock ownership in AxioMed, DePuy, OrthoMEMS, and Turning Point; consulting for AxioMed; and research support from OREF and Rawlings. Dr. Chao received an honorarium from Varian Medical Systems.

\section{Author Contributions}

Conception and design: Miller, Balagamwala. Acquisition of data: Miller. Analysis and interpretation of data: Miller. Drafting the article: Miller, Balagamwala. Critically revising the article: all authors. Reviewed submitted version of manuscript: all authors. Approved the final version of the manuscript on behalf of all authors: Chao. Statistical analysis: Miller. Study supervision: Suh.

\section{Supplemental Information}

Previous Presentations

Portions of this work were presented in abstract form at the 2016 Annual Meeting of the American Society for Radiation Oncology, September 25-28, 2016.

\section{Correspondence}

Samuel T. Chao, Department of Radiation Oncology, Cleveland Clinic, 9500 Euclid Ave., Desk T28, Cleveland, OH 44195. email: chaos@ccf.org. 